the freces of children receiving full doses of dimol four-hourly.

6. Owing to the small number of cases treated, it is impossible to draw any wide conclusions. 'The results, however, appear to justify further investigation, and " are given in the hope that other observers may give the treatment a trial and report their results.

7. I have only to add that I have no interest whatever, financial or otherwise, in the preparation or sale of dimol.

\section{ON THE TREATMENT OF \\ NON-MALIGNANT AFFECTIONS OF THE COLON.}

BY SIR W. ARBUTHNO'T LANE, BAR'T, M.S. LoND., F.R.C.S. ENG.

Whes I was asked to open this discussion* I willingly acquiesced, though I felt the honour was greater than I was able to do justice to.

It was formerly customary to regard the several abnormal conditions of the colon as separate entities or diseases. Personally, I do not accept this situation, but consider them to be one and all the direct or indirect results of chronic intestinal stasis. In order to substantiate the accuracy and correctness of $\mathrm{my}$ views I must first draw your attention to the two great groups of conditions which I found in the living abdomen-namely, those cases of intestinal stagnation in which a reaction takes place and those in which it is altogether absent.

Group Characterised by the Formation of Bands.

In the vigorous subject delay of the accumulated contents in the pelvic colon leads to the formation of acquired bands on the under surface of the mesentery securing the junction of the iliac and the pelvic segments of the colon. The mode of formation of these, as of all the other acquired bands which develop to hold up and secure a loaded colon, is very simple. They commence as streaks on the surface of the mesentery, starting from its base and encroaching gradually along its length. After a time they reach the wall of the intestine. The streaks soon become a distinct membrane which, at a later period, may be separated from the mesentery except at its base, and at its terminal extremities. This membrane tends to contract and to drag the bowel towards the iliac fossa, where it finally becomes firmly fixed. The fibres that grip the colon not only diminish its lumen by angulating it, but they also tend to reduce its calibre still further by rotating it on its long axis.

Like most of the efforts that nature makes to help the individual to bear a useful relationship to his surroundings, this membrane serves a beneficial purpose in the first instance, tending to support the loaded bowel, and so opposing its elongation and prolapse. Later this originally useful structure tends by its contraction to obstruct the lumen of the bowel and to place the life of the individual in serious peril. This membrane in its development is very liable to grip the ovary and Fallopian tube and to fix them immovably on the iliac fossa. It may interfere with the function of the Fallopian tube impeding the passage of the ovum through it and causing extra-uterine foetation, or it may prevent the entry of the ovum and so render the patient sterile on the left side. Besides securing and fixing the ovary it sooner or later surrounds it, and causes it to undergo a cystic change. Later the degenerating ovary increases in size, bursts through the surrounding membrane, and forms a mobile ovarian tumour.

Gynæcologists are only too familiar with the pain which women experience in the left iliac fossa and which is so often exaggerated by the approach of the period in the struggle of the bowel to force fæcal matter through the anchored and obstructed colon

* The discussion took place in the Section of Surgery of the $*$ The discussion took place in the Section of Surgery of the
British Medical Association at Glasgow on July 28 th, 1922 . over the sensitive ovary gorged with blood. These conditions have been referred to fully in a paper by Mr. II. Chapple. ${ }^{1}$ 'To this particular acquired band and to its effect on the colon I gave the name of the "first and last kink." The first because it is the earliest of the acquired membranes to develop and the last because it is the lowest in the drainage scheme.

This development of a band controlling the lumen of the lower portion of the bowel is, perhaps, the most important acquired change in the whole body since

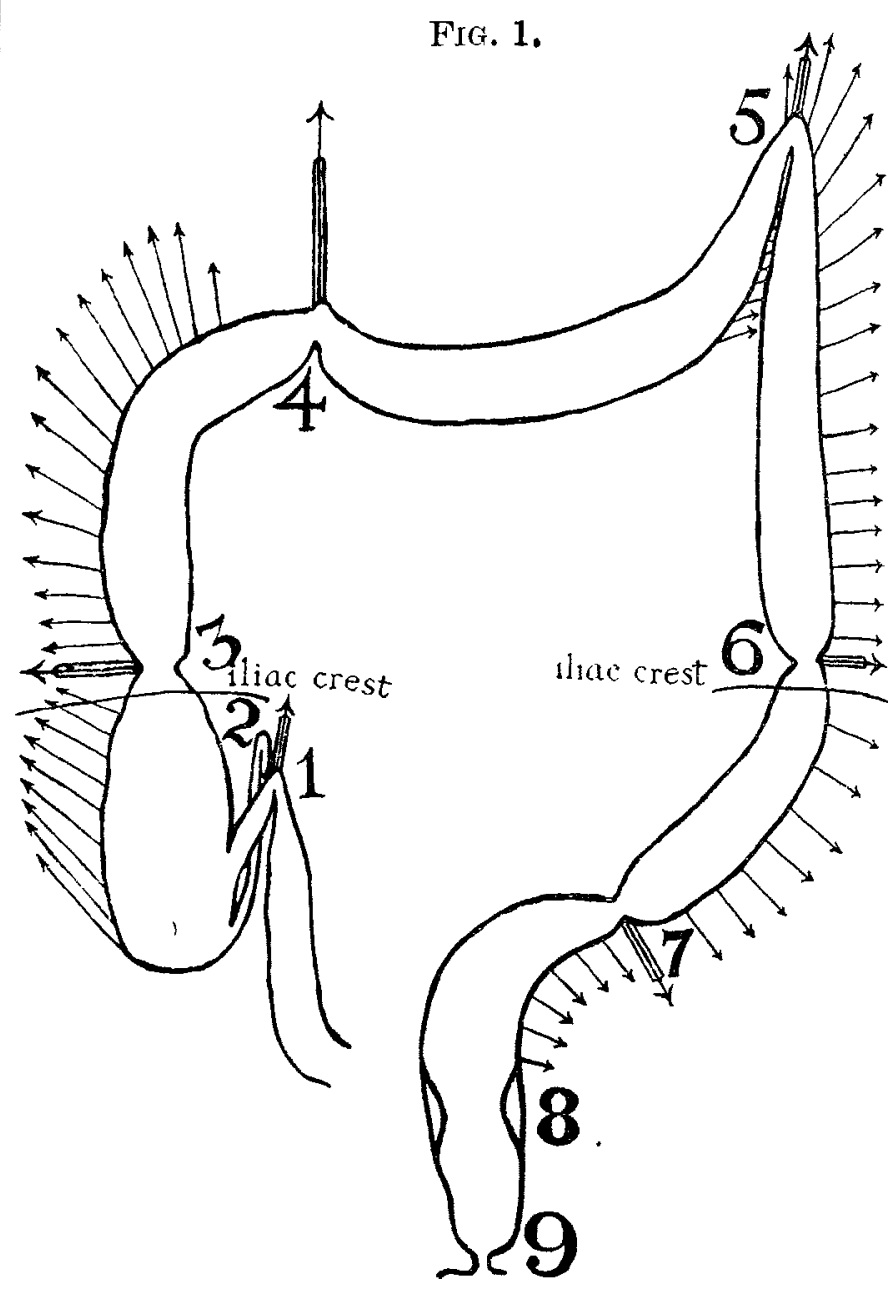

The arrows show the direction of the lines of resistance. 1. Indicates the ileal sink. 2 . The appendix passing up behind the ileum and controlling its lumen in the erect posture (it is secured by evolutionary bands to the back of the mesentery and is itself kinked and its distal portion obstructed by the anchoring adhesions). 3. The strong band which obstructs the Iumen of this portion of the colon. 4. The band which extends down from the liver, gall-bladder, pylorus, and duodenum. 5. 'The splenic flexure, whose angularity is accentuated and whose lumen is correspondingly diminished. 6 . The deformity of the descending colon by a band similar to 3.7 . The last kink. 8. The circular band of muscle fibre described by W. J. Mayo. 9 . The internal sphincter.

it is the primary cause of a vast amount of subsequent trouble in the gastro-intestinal tract and elsewhere. Its immediate effect in producing an obstruction at this point is to render the partially occluded colon a very common seat of cancer, while in fat subjects the bowel proximal to it is a very usual site of diverticulitis, the causation and pathology of which I described fully in the Guy's Hospital Reports, 1885, in a paper entitled Pathology of Extravasation of Urine. An abstract of this was published in THE LANCET, vol. i., 1920, p. 220. The indirect consequence of the obstruction to the passage of fæcal matter through the first and last kink is the accumulation of material in the whole length of the colon. The increased load in the obstructed bowel results in the development of precisely the same type of acquired membrane on the outer layer of peritoneum which extends from the convexity of the large bowel to the abdominal wall. It serves to fix the colon and to prevent its elongation and prolapse. It is most marked in certain positions where the consequent construction of the lumen of the bowel may produce inflammatory or cancerous changes.

1 Guy's Hospital Gazette, 1921, vol. xxxv, p. 164. 
The localities so affected are immediately above the iliac crests, at the splenic flexure, below the gallbladder, where an acquired band from the liver, gallbladder, duodenum, and pylorus descends to hold up and later obstruct the transverse colon, over the outer aspect of the cacum, where it has recently been called Jackson's membrane, and again on the under surface of the terminal mesentery of the small intestine, which, with the end of the ileum, support the cæcum, forming its internal lateral ligament. In America this band has been given my name and is called "Lane's kink." These several acquired bands are illustrated in Fig. 1. I described these in detail more than twenty years ago. Evolutionary Origin, Function, and Structure of Bands. What I want particularly to call attention to is that these so-called separate bands are all evolutionary and identical in origin, function, and structure, and are due to the effort of the organism to meet an abnormal loading up of the bowel because of a distal obstruction, and to enable it to perform its function to the best of its capacity and not to prolapse. As I have pointed out, the initial purpose of their existence is carried out efficiently for a time, but later the continued contraction of the newly-formed membranes results in obstruction of the lumen of the bowel and in consequent disease. This is usually most marked at the ileal kink (as I call the one named after me) and at the splenic flexure. Too much attention cannot be paid to the mechanism of these acquired membranes and to the effect they exert both immediately and secondarily upon the functioning of the large bowel. When I first described them their existence was altogether denied. Later when they were found to be only too obvious the tendency was to regard them as separate and independent structures, to argue as to whether they were congenital or inflammatory in origin, or to assert that they have no influence whatever upon the normal function of the intestine. Mr. Chapple carefully examined 52 newly-born foetuses in Guy's Hospital and could find no trace of bands similar to those I described in the adult. This shows that they are obviously not congenital. No one familiar with the appearance, structure, and mode of development of the bands in the living subject could regard them as inflammatory. On the other hand, anyone who has studied the several changes which the skeleton and soft parts undergo in the varying mechanical relationship of the individual to his surroundings can have no doubt as to their evolutionary origin.

\section{"The Controlling Appendix."}

Another important development, which arises not infrequently in consequence of the strain exerted by the loaded coecum, is what I called "the controlling appendix." It is one of very great pathological and clinical importance. This, like the ileal kink, has now been pretty generally accepted by surgeons. Instead of developing a membrane on the under surface of the mesentery of the terminal ileum the appendix is employed in the novel function of a ligament. It becomes attached by acquired adhesions to the under surface of the mesentery, and therefore it lies behind the end of the ileum. By the gradual contraction of the acquired adhesions it is rendered progressively tighter. Its situation is such that when the ileum and cxcum drop into the pelvis the ileum is kinked abruptly over the anchored appendix, and its lumen is more or less occluded in consequence, like that of an inflated tyre hung over a hook. This controlling appendix, or the equally damaging ileal kink, are responsible for very much of the ileal stasis and infection consequent on their presence and which play such an important part in the sequence of chronic intestinal stasis. The kinking of the anchored appendix itself renders its own lumen liable to become obstructed. Consequently inflammation follows, and this attracts attention to it. The removal of such an anchored appendix is followed by great benefit to the patient.

It is in this type of static colon that cancer of some portion of the gastro-intestinal tract commonly occurs, since the factors that determine cancer and which are simply mechanical are present in a more or less marked degree. Many of the patients are robust and are regarded as healthy people by the casual observer.

Group Characterised by Absence of Bands.

The other extreme type of chronic intestinal stasis is characterised by a complete want of effort on the part of the organism to oppose the elongation and prolapse of the large bowel, no new acquired bands or membranes being formed. The pelvic colon becomes greatly elongated, and, puddling in the pelvis, this long, loose bowel forms many abrupt angles and offers a great obstacle to the passage of solid material through it since the more the individual strains to expel the bowel contents the further is the flaccid gut driven into the lower limit of the pelvis. While in the type first described the acquired band forming the first and last kink constitutes the chief obstacle to the emptying of the colon; in this type obstruction is due to the great elongation and prolapse of the pelvic colon, so that the mechanical conditions are perfectly distinct in the two extremes. (See Fig. 2.)

Fig. 2.

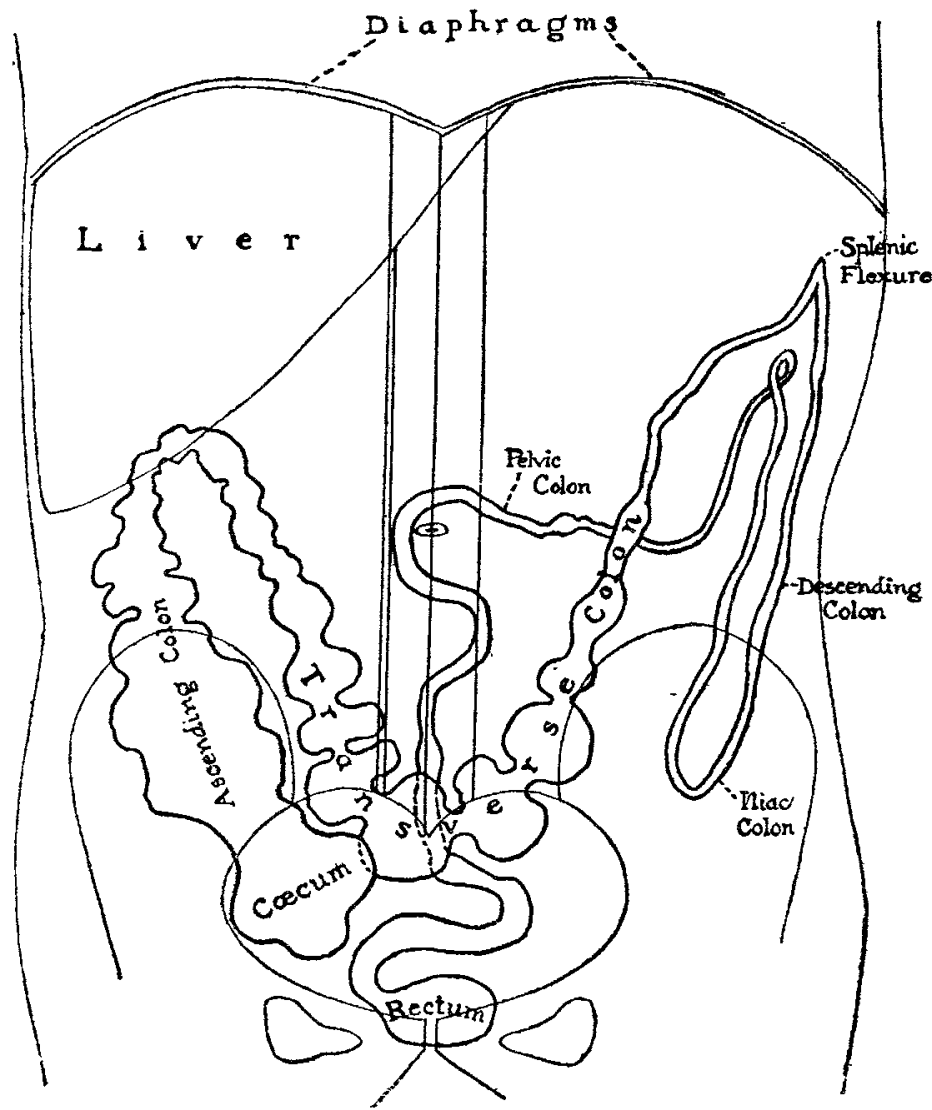

Diagram, by Dr. Jordan, showing the radiographic appearances of an extreme case of chronic intestinal stasis. It was taken while the patient was in the recumbent posture. It is sufficiently clear to require no further detailed description.

Following on this obstruction the proximal colon elongates to a great extent and prolapses. As far as I know the mere prolapse of the rest of the larye bowel in no way affects its function, except in the case of the cæcum, when it may twist the end of the ileum and obstruct its effluent. ${ }^{2}$ This condition of the cæecum and ascending colon may be greatly benefited by plicating the bowel or by the method of anchoring advocated so strongly by Mr. George Waugh. Curiously enough, the prolapse of the large bowel-or, as it is technically called, enteroptosis-has monopolised the attention of most operators, who attempt to rolieve the symptoms of the patient by fastening the bowel in position or by excising portions of the elongated transverse colon. I have seen no permanent advantage from such operations in patients on whom I have operated subsequently, since such conditions are obviously secondary and not primary. In consequence of the obstruction to the passage of the intestinal contents, which results from the elongation of the pelvic colon, an infection of the mucous membrane

This was described and demonstrated in a remarkable manner by Dr. A. C. Jordan. 
of the proximal colon takes place, producing a condition called colitis. This infertion causes a spasm of the muscle wall of the colon and a consequent reduction of the lumen of the inflamed bowel, so producing a very serious mechanical obstacle to the passage of material through it. As I have previously indicated the mechanical conditions are perfectly dissimilar in the two extreme varieties of stasis, while between the two extremes every variety of degrce and combination exists.

In this typs there is no point of constriction at which hard faces can be definitely obstructed, and therefore no area of impact where cancer is likely to develop. For this reason cancer of the colon is not seen as a sequel in this form of stasis, though it is common in parts of the body other than the gastro-intestinal tract. This I have discussed fully in many previous communications. I feel that it is most important from the point of view of treatment, medical as well as surgical, that we should render ourselves perfectly familiar with the exaggerated types I have described and with the intermediate varieties. ${ }^{3}$

\section{Operative Interference in the Type Characterised by the} Formation of Bands.

In the first extreme type of stasis the conditions which call for operative interference on the colon apart from cancer are the obstruction caused by acquired bands, by far the most important of which is that which produces the first and lost kink.

The operation that I perform with great frequency is the careful separation of the bands which form this kink and the accurate apposition of the peritoneal edges should any surface be left deprived of its serous covering. Other controlling bands are sought for, and if present are divided. The termination of the ileum is examined and, should a controlling appendix or an ileal kink be present, these are dealt with, and any raw surfaces carefully covered in by peritoneum. If the membrane forming the ileal kink is at all extensive it is important, after freeing it, to leave a drainagetube in position, for the lymphatics which are divided in the acquired membrane contain septic organisms which, if not drained, readily escape and set up a local peritonitis which may terminate fatally.

The presence of such septic organisms in the area from which the acquired membrane was removed have been demonstrated by cultures made by Dr. Nathan Mutch at the time of operation. This fact accounts for many cases of unexplained fatal peritonitis in operations on the appendix. I may say that I go through this sequence most systematically when dealing with any of the sequelæ of stasis, such as gastric and duodenal ulcer, gall-stones, \&c.

The other but very much less frequent complication in this type in fat subjects is diverticulitis. This usually occurs in the area of bowel proximal to the last kink, or it may develop in any portion of the large bowel behind an obstruction. If still definitely non-malignant the most simple method of dealing with this diverticular formation in fat subjects consequent on obstruction is to put the divided end of the ileum into the pelvic colon. In this case, as in that of colectomy, the patient should be instructed to secure three evacuations daily. By such means any regurgitation of the contents of the ileum into the colon proximal to the junction can be readily avoided. Occasionally it is advisable to excise the diseased area and to unite the proximal bowel to the pelvic colon. 'This must depend on the possibility of mobilising the bowel and upon the nature of the intestinal wall. In some cases a colectomy offers the best results.

Another complication of this type of stasis is tuberculous ulceration of the colon which is frequently limited to its proximal half. For this a complete colectomy may be performed, or, if the condition of the patient renders it advisable to limit the extent of the operation as much as possible, the ileum may be divided and be put into the pelvic colon and the colon

- Reflections on the Evolution of Disease, THe LANoEr, Dec. 20th, 1919. Cancer of the Colon: its Causation and Treatment, THE LANCET, Dec, $11 \mathrm{th}, 1920$. excised beyond the limit of the area affected by tubercle. What procedure is adopted must vary with the conditions present at the operation.

It is the opinion of certain experts in tropical diseases that dysentery only becomes chronic in those affected by intestinal stasis, and it has been demonstrated by Mr. J. W. Jackson in a most interesting communication ${ }^{4}$ that chronic amobic dysentery can be frequently cured by freeing the intestine from obstruction by band or controlling appendix.

Operative Interference in the Group Characterised by Absence of Bands.

In the second type of static colon the mechanical difficulties are, first, the elongated pelvic colon, and, second, the obstruction consequent on the spasm of the muscle coat of the bowel produced by the inflam. mation of its mucous lining. The symptoms which. call for operation in this type are not due so much. to the pain and constipation or diarrhoea but to the auto-intoxication which is the result of infection of the contents of the small intestine, which produces most serious degenerative processes in every tissue in the body and renders the subject liable to infection by other diseases, as rheumatoid arthritis, tubercle, \&c.

It would seem that the simplest operative procedure would be to excise the surplus pelvic colon and to restore it to its normal length; such a measure is beneficial in certain circumstances, but in the majority of cases the best results can be obtained by a. colectomy or by dividing the ileum and putting it into the pelvic colon, subsequently taking the precautions to secure the frequent evacuation of the pelvic colon which have already been indicated. Needless to say, no operation should be considered till every medical means have been taken to overcome the constipation, to allay the spasm of the muscle of the bowel by curing the colitis, and to free the ileal effluent.

I have described the operations of ileo-colostomy and of colectomy so frequently that it is unnecessary to repeat the descriptions again in detail. I would merely point out that in this type, as opposed to the other, colectomy is rendered very easy because of the prolapse and elongation of the colon and by the absence of any retaining membranes.

A complication of the elongated large bowel which increase the obstacle to the passage of its contents is a twist. It is usually chronic but owing to the abrupt exaggeration of the twist it may become acute and produce a volvulus. In certain cases I have excised the volvulus and in others I have performed a colectomy. The same treatment applies equally to the congenital enlargement of the colon, sometimes called megacolon. As regards ulcerative colitis, when medical treatment has failed to cure the condition, the only operation which has been of any real service in my experience has been a colectomy. This I have performed with complete success very early in childhood. Even after a colectomy prolonged local and vaccine treatment for a considerable period is usually called for, to get rid of the residual ulceration which so often exists in the rectum and in the portion of pelvic colon which has necessarily been left in the body. Much the same operative treatment applies to severe cases of mucous and membranous colitis, but it is resorted to much less frequently. Before leaving the subject of these operative procedures I would urge the importance of attempting to avoid the sequence of conditions which result from stasis and which produce so much misery and death. Cancer is the final stage in the sequence of chronic intestinal stasis. It is the last chapter in the story of defective drainage of the large bowel as it is in the rest of the gastro-intestinal tract. Its treatment has been excluded from consideration in this discussion. Another effect of infection. of the badly drained colon is inflammation of the appendix. On the treatment of this complication we are in general agreement.

I cannot impress on you too strongly Colonel $R$. MeCarrison's experience in India. He spent nine years. in the Himalayas, during which time he had a large

- Medical Press and Circular, May 3rd, 1922. 
practice, performing more than 400 capital operations each year. During that time he never saw one case of asthenic dyspepsia, of gastric or duodenal ulcer, of appendicitis, of mucous colitis, or of cancer-all evidences of chronic intestinal stasis in their normal sequence. I have obtained precisely similar evidence from other very experienced medical men who have been engaged in practice for many years among the primitive races. It is clearly our duty not so much to devise new operations as to attempt to prevent the diseases for which so many operations are done. This is the biggest problem before the profession at the present moment, and it is one that must be solved.

\section{THE TREATMEN'T OF}

\section{ACUTE OBSTRUCTION FROM CANCER OF THE COLON.}

By J. P. LOCKHART-MUMMERY, F.R.C.S. ENG., SENIOR SURGEON, ST. MARK'S HOSPITAL FOR DISEASES OF TIIE RECTUM, LONDON, E.C.

IT is now a well-recognised surgical principle that no attempt should be made to resect the bowel when obstruction is present, and that the proper treatment when acute obstruction exists is to be content with draining the bowel by the establishment of an artificial opening, the removal of the cause of the obstruction being deferred till later, when the patient has recovered from the acute symptoms. While this is now the more or less general practice, most surgeons still start by doing an exploratory laparotomy, presumably with a view of finding the cause before attempting to deal with the obstruction. This often results in the surgeon getting into difficulties owing to the distended coils of bowel, which are liable to escape from the wound and be difficult to replace, and to the danger of the bowel splitting from the tension. Few operations, in fact, give rise to such serious difficulties as an exploratory laparotomy on a patient with acute obstruction of the colon.

It seems to me that there is no necessity for, or object in, the performance of an exploratory laparotomy at all in the acute stage, and that a much better plan is simply to drain the cæcum and leave the exploration till the patient has recovered from the obstruction, when a resection of the growth can be performed with reasonable safety. It is generally admitted that cæcostomy is the best method of dealing with an obstructed colon ; it can be performed through a small incision with none of the risks attached to an exploratory operation. Moreover, the presence of a cxcal fistula will render any subsequent resection of the colon much safer, and the opening is as far as possible away from the situation of the exploratory wound in the abdominal wall, assuming that this is, as usual, on the left side of the abdomen. The only objections that have been urged against a cæcostomy as opposed to a colostomy in these cases are that owing to the liquid nature of the contents of the cæcum such openings are difficult to control and may be difficult to close.

The following method which I have been using lately appears to overcome both these difficulties. $A$ small incision is made over the cæcum and a knuckle of the creal wall drawn out and protected with gauze swabs. A hole is made in the cacal wall with a knife, and a rubber drainage-tube about half an inch in diameter is pushed into the cæcum for two inches. The tube is then stitched to the cæcal wall with catgut, each stitch going through the wall of the tube and picking up the cacal wall half an inch away from the tube. When these are tied a cuff of cæcal wall will have been turned in. A purse-string suture is now inserted well away from the tube and tied so as to turn in more of the cæcal wall. The ends of the suture, after being tied, are brought through the deep fascia and peritoneum, and one or two other sutures placed so as to fix the cæcum at the point at which the tube enters to the deep surface of the abdominal wall. The abdomen is then sewn up around the tube. wide collapsible rubber tube is tied on to the end of the rubber drainage-tube projecting from the dressings, and is carried over into a pail under the bed. The tube remains quite water-tight and gas-tight for as long as a week, and as the contents of the cæcum are liquid they drain quite readily through a tube of this size; no leakage takes place in the wound and the patient can be kept dry and comfortable.

After the obstruction has been relieved, and when the patient has lost all signs of toxæmia-which will generally be in about five or six days-the abdomen can be opened in the mid-line, or on the left side, and the cause of the obstruction ascertained and dealt with under the most favourable circumstances. The presence of a cæcostomy renders any such operation as resection of part of the colon much easier and safer, and the absence of toxæmia and distension greatly reduces the risks. When the tube in the cæcum becomes loose, which is usually in about eight or nine days, it may be removed, and as a rule there will
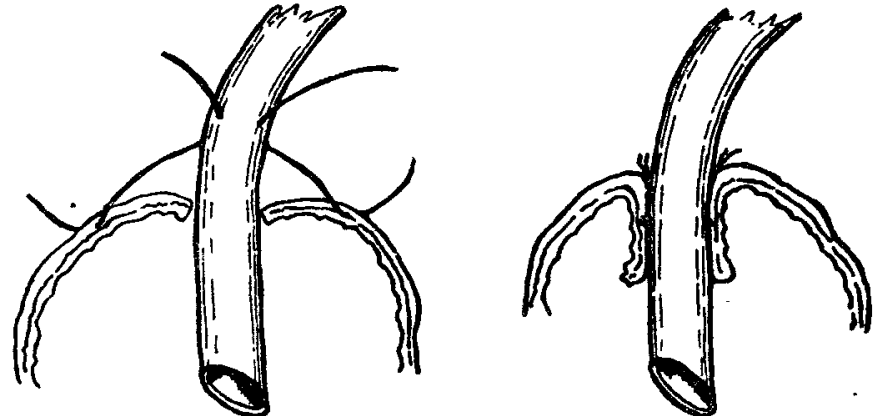

Diagram to show method of inserting stitches in performing cæcostomy.

only be slight leakage for a few days, assuming that the original obstruction has been removed, or that a colostomy has been done above it.

I have never known an opening made in this way fail to close spontaneously in a week or ten days, and most of them have not leaked after the tube has come away. I believe this to be a much safer method of dealing with cases of acute obstruction of the large bowel than exploration of the abdomen, and I think it is the right method when one can be reasonably sure that the obstruction is due to a growth. In sudden obstruction, without any previous history, one would not be able, as a rule, to exclude a volvulus, and exploration is called for, but in many cases one can be reasonably sure that volvulus or hernia is not the causal agent; in such instances a cæcostomy without exploration of the abdomen will save more lives than are likely to be lost by the missing of some obscure hernia of the colon.

If, at the operation, the cæcum is not found to be distended exploration is, of course, called for as the obstruction must be in the small gut. Chronic intussusception in an adult might be confused with a growth in the colon, but this is not likely if the history of the case is known, and further cæcostomy will, in most cases, by relieving the obstruction, prevent gangrene of the intussusception, so that the treatment would be effectual.

A NOTE ON

\section{RECUPERATIVE RAY BATHS.}

\section{By EDWARD JAMES DECK, M.R.C.S. ENG.}

I SHOULD like to draw attention to the beneficial results to be obtained by exposing the whole body to multiple arcs giving forth rays in which the ultra violet predominates. The method of producing this rich volume of rays is the electrical combustion of practically pure electrodes, containing 99 per cent. of tungsten.

The cases I have selected to demonstrate the value of this treatment are, I think, appropriate because of their severity, each case having been definitely diagnosed as malignant disease, attended with a marked degree of anæmia besides other general 